\title{
Electron Interference in Ballistic Graphene Nanoconstrictions
}

Baringhaus, Jens; Settnes, Mikkel; Aprojanz, Johannes; Power, Stephen; Jauho, Antti-Pekka; Tegenkamp, Christoph

Published in:

Physical Review Letters

Link to article, DOI:

10.1103/PhysRevLett.116.186602

Publication date:

2016

Document Version

Publisher's PDF, also known as Version of record

Link back to DTU Orbit

Citation (APA):

Baringhaus, J., Settnes, M., Aprojanz, J., Power, S., Jauho, A-P., \& Tegenkamp, C. (2016). Electron Interference in Ballistic Graphene Nanoconstrictions. Physical Review Letters, 116(18), [186602]. https://doi.org/10.1103/PhysRevLett.116.186602

\section{General rights}

Copyright and moral rights for the publications made accessible in the public portal are retained by the authors and/or other copyright owners and it is a condition of accessing publications that users recognise and abide by the legal requirements associated with these rights.

- Users may download and print one copy of any publication from the public portal for the purpose of private study or research.

- You may not further distribute the material or use it for any profit-making activity or commercial gain

- You may freely distribute the URL identifying the publication in the public portal

If you believe that this document breaches copyright please contact us providing details, and we will remove access to the work immediately and investigate your claim 


\title{
Electron Interference in Ballistic Graphene Nanoconstrictions
}

\author{
Jens Baringhaus, ${ }^{1}$ Mikkel Settnes, ${ }^{3}$ Johannes Aprojanz, ${ }^{1}$ Stephen R. Power, ${ }^{3}$ \\ Antti-Pekka Jauho, ${ }^{3}$ and Christoph Tegenkamp ${ }^{1,2, *}$ \\ ${ }^{1}$ Institut für Festkörperphysik, Leibniz Universität Hannover, Appelstraße 2, 30167 Hannover, Germany \\ ${ }^{2}$ Laboratory of Nano and Quantum Engineering (LNQE), Leibniz Universität Hannover, \\ Schneiderberg 39, 30167 Hannover, Germany \\ ${ }^{3}$ Center for Nanostructured Graphene (CNG), DTU Nanotech, Technical University of Denmark, 2800 Kgs. Lyngby, Denmark
}

(Received 6 January 2016; published 5 May 2016)

\begin{abstract}
We realize nanometer size constrictions in ballistic graphene nanoribbons grown on sidewalls of $\mathrm{SiC}$ mesa structures. The high quality of our devices allows the observation of a number of electronic quantum interference phenomena. The transmissions of Fabry-Perot-like resonances are probed by in situ transport measurements at various temperatures. The energies of the resonances are determined by the size of the constrictions, which can be controlled precisely using STM lithography. The temperature and size dependence of the measured conductances are in quantitative agreement with tight-binding calculations. The fact that these interference effects are visible even at room temperature makes the reported devices attractive as building blocks for future carbon based electronics.
\end{abstract}

DOI: 10.1103/PhysRevLett.116.186602

Graphene nanoribbons (GNRs) are an ideal system to study electronic transport phenomena in the coherent regime due to the extremely long mean free path and coherence length of charge carriers [1-3]. In analogy with subwavelength optics, the coherent transmission of electrons through narrow constrictions within such ballistic ribbons gives rise to interference phenomena [4,5]. Graphene nanoconstrictions (GNCs) are an important building block in carbon electronics, especially for valleytronic applications [6], and hence their atomically precise synthesis as well as electronic characterization is of great importance [7-10]. Unfortunately, lithographically defined GNCs and GNRs exhibit rather rough edges and the inherent defect potentials limit drastically the achievable mean free paths $[11,12]$.

The growth of graphene on the sidewalls of $\mathrm{SiC}$ mesa structures was reported to produce graphene nanostructures of exceptionally high quality [3,13-19]. Their hallmark feature is the ballistic transport of electrons, which can be observed on micrometer length scales $[3,13]$. The robustness of the ballistic behavior makes these devices a prime platform for studying interference phenomena at graphene interfaces. For the patterning of narrow constrictions into the sidewall ribbon, STM lithography is the method of choice. It was shown to cut graphene sheets with atomic precision while preserving the quality of the pristine material away from the cut $[20,21]$.

For the growth of GNRs we use $\mathrm{SiC}$ wafers commercially purchased from SiCrystal AG. SiC substrates were flattened by using the face-to-face heating method $[13,22]$ and subsequently mesa structures with lateral dimensions between 1 and $8 \mu \mathrm{m}$ and a height of $20 \mathrm{~nm}$ were defined by using standard UV lithography combined with reactive ion etching (gas mixture $20 / 7 \mathrm{SF}_{6} / \mathrm{O}_{2}$, power $30 \mathrm{~W}$ ).
GNRs were grown exclusively on the sidewall of the mesa following standard recipes $[13,16]$.

A four-tip STM in combination with a high-resolution SEM is used for both transport experiments and in situ patterning of the nanoribbons. The local characterization of graphene and ballistic sidewall graphene nanoribbons with multiple STM probes is nondestructive and highly controlled $[3,13,23]$ and offers a unique possibility to study directional transport effects in graphene nanostructures [24]. Further details about the experiments as well as the theoretical modeling are explained in the Supplemental Material [25].

The most intriguing feature of the ballistic sidewall ribbons is a probe-spacing and temperature independent conductance of $1 e^{2} / h$ [3], which indicates single-channel transport. In such a ballistic ribbon, abrupt graphene interfaces can be introduced in the form of a narrow, a few nanometers wide and long, constriction. For this purpose, a STM tip is navigated across the graphene covered sidewall under extreme tunneling conditions, i.e., at large bias voltages and tunneling currents of about $V_{t}<-5 \mathrm{~V}$ and $I_{t} \geq 50 \mathrm{nA}$. The graphene underneath the STM tip is thereby removed (cf. Ref. [25], Fig. 1). The underlying etching mechanism is not fully understood, but relies most likely on the local breaking of carbon-carbon bonds underneath the STM tip via field-emitted electrons $[37,38]$. The tip was always moved transversely over the ribbon starting at the trench and ending on the plateau of the mesa structure as shown schematically in Fig. 1. This results in the formation of a constriction at the lower edge of the ribbon as verified by a subsequent STM characterization. For etching away the graphene at the lower ribbon edge, much higher etching voltages are needed. This is 


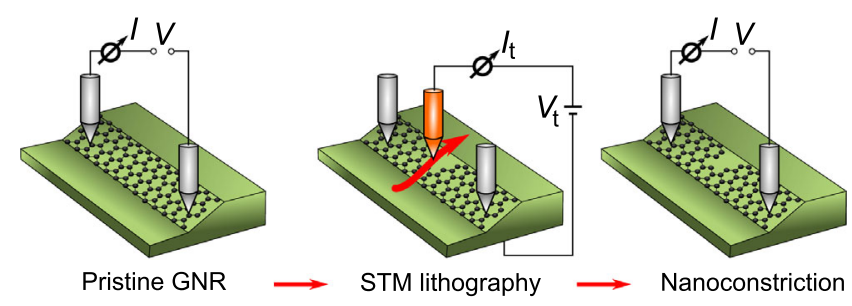

FIG. 1. Synthesis of sidewall GNCs by STM lithography. First, a reference two-point-probe measurement is performed on the pristine sidewall GNR to ensure the presence of a ballistic channel. The GNC is subsequently defined into the GNR via local etching by means of a STM tip. A second two-point measurement probes the transport properties of the GNC.

most likely due to the different geometry of this edge, which terminates almost vertically into the substrate $[3,39]$.

A schematic view of the graphene nanoconstriction obtained by STM lithography is given in Fig. 2(a). The corresponding STM images of characteristic positions along the cut are displayed in Fig. 2(b), showing the lower ribbon edge, the central part, and the upper edge. The graphene appears bright compared to the underlying substrate. Obviously, the sidewall GNR was cut through at the upper edge to the mesa plateau and the middle of the ribbon, but not at the lower edge to the trench. Here, a small patch of graphene remains, forming a narrow constriction with lateral dimensions of only a few nanometers. Atomically resolved STM images ensure that the graphene lattice in the vicinity of the cut was not damaged by the cutting procedure as shown in Fig. 2(c). The local density of states (LDOS), obtained by scanning tunneling spectroscopy, further supports these findings. Tunneling spectra, Fig. 2(d), were taken directly on the cut and on the surrounding graphene [Fig. 2(c), middle frame, red and black dots, respectively]. The LDOS of the intact graphene surrounding the cut exhibits the characteristic $V$ shape, with a tunneling conductance minimum precisely at $0 \mathrm{~V}$. Hence, the charge neutrality of the sidewall ribbon is preserved $[3,13]$. On the other hand, the LDOS recorded on the cut drops to zero for the tunneling bias voltage range $-0.50<V_{t}<0.25 \mathrm{~V}$. Hence, an electronic current flowing from left to right has to be transmitted through the constriction.

The electronic transport through the sidewall GNC was recorded by biasing the constriction with two tips placed in Ohmic contact on the sidewall GNR with the GNC in between (as schematically indicated in Fig. 1). The $I V$ characteristics of a biased sidewall GNC $(L=6 \mathrm{~nm}$, $W=2 \mathrm{~nm}$ ) recorded at different temperatures in the range from 28 to $300 \mathrm{~K}$ are shown in Fig. 3(a) together with a reference measurement of the pristine, unpatterned sidewall GNR. The pristine ribbon exhibits a completely linear $I V$ curve with a conductance of $1 e^{2} / h$, which is characteristic for ballistic transport in a fully nondegenerate channel [3]. In contrast, the $I V$ curves through the constrictions are
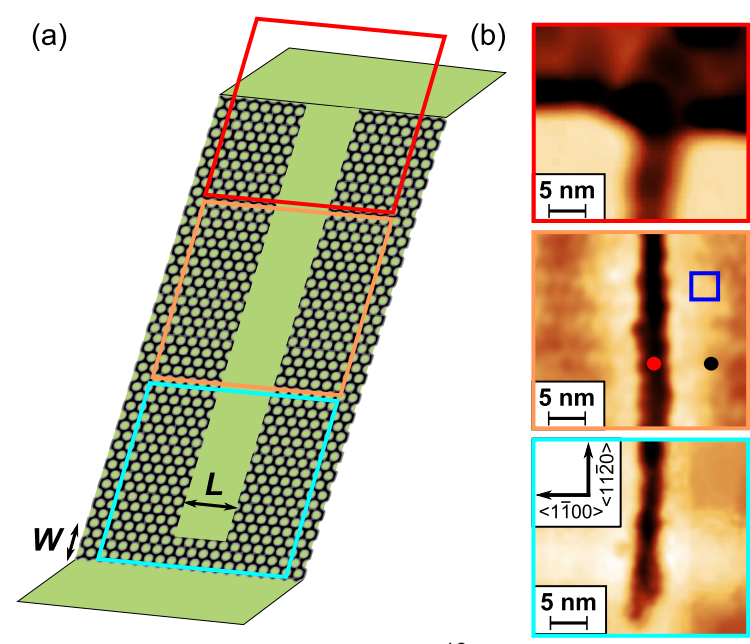

(c)

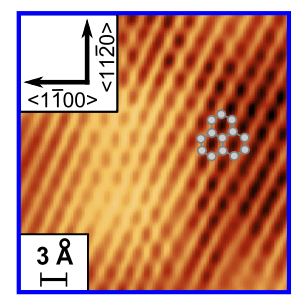

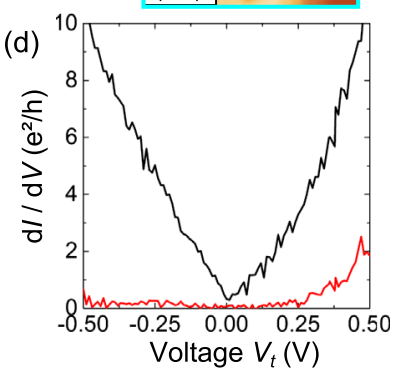

FIG. 2. STM study of a sidewall GNC. (a) Schematic view of the graphene structure on the $\mathrm{SiC}$ mesa sidewall after synthesis of a narrow constriction with STM lithography. (b) STM images of the top, center, and bottom part of the cut (tip voltage for imaging $V_{t}=3 \mathrm{~V}$, tunneling current $I_{t}=1 \mathrm{nA}$ ). In the bottom frame, the presence of a GNC is confirmed. The red and black dots in the middle frame indicate the locations at which spectroscopy was performed, panel (d). (c) Atomically resolved STM topograph of the graphene lattice in the vicinity of the cut [see blue rectangle in (b)] $\left(V_{t}=200 \mathrm{mV}, I_{t}=100 \mathrm{pA}\right)$. (d) $d I_{t} / d V_{t}$ spectroscopy of the GNC. Red curve: spectrum acquired directly on the cut [openfeedback parameters (setpoint): $V_{t}=0.2 \mathrm{~V}, I_{t}=0.2 \mathrm{nA}$, modulation voltage $\left.V_{\mathrm{rms}}=15 \mathrm{mV}\right]$. Black curve: spectrum acquired in the vicinity of the cut on the unpatterned graphene lattice $\left(V_{t}=0.4 \mathrm{~V}, I_{t}=0.4 \mathrm{nA}\right.$, modulation voltage $\left.V_{\mathrm{rms}}=20 \mathrm{mV}\right)$.

clearly nonlinear. They can be well described by the phenomenological Kaiser expression [40] (details are given in Ref. [25], Sec. II), which is frequently used to describe nonlinearities in the $I V$ curves for carbon nanotubes. In the low-bias regime, the opening of a small transport gap $(\Delta \approx 10 \mathrm{meV})$ is clearly visible. For $T=28 \mathrm{~K}$ the zerobias conductance drops to zero. With increasing temperature, the slope around zero bias increases while for $V_{b}>10 \mathrm{meV}$ it remains almost constant throughout the whole temperature range.

More insight can be gained from the differential conductance displayed in Fig. 3(b). The $d I / d V$ curves were obtained by either the numerical differentiation of the $I V$ curves and averaging over at least 50 individual measurements, or directly by using standard low-frequency lock-in techniques. The results of both methods agree well with 
(a)

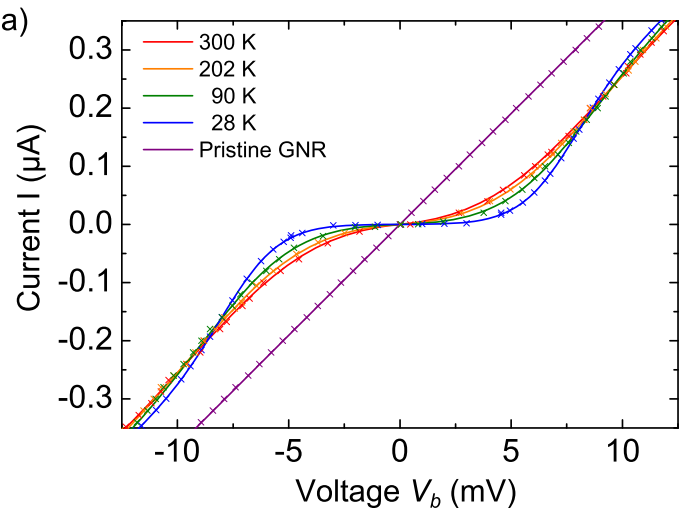

(c)

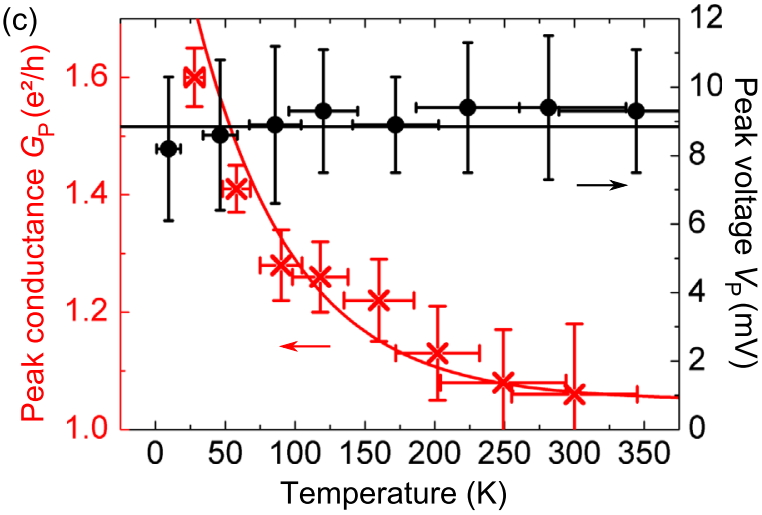

(b)

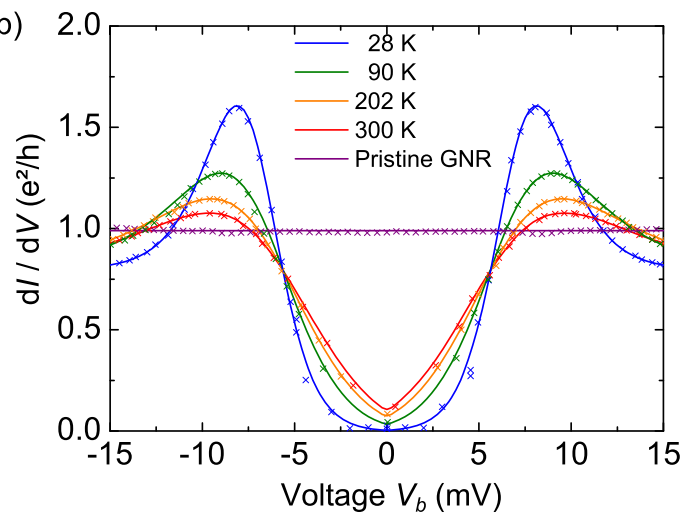

(d)

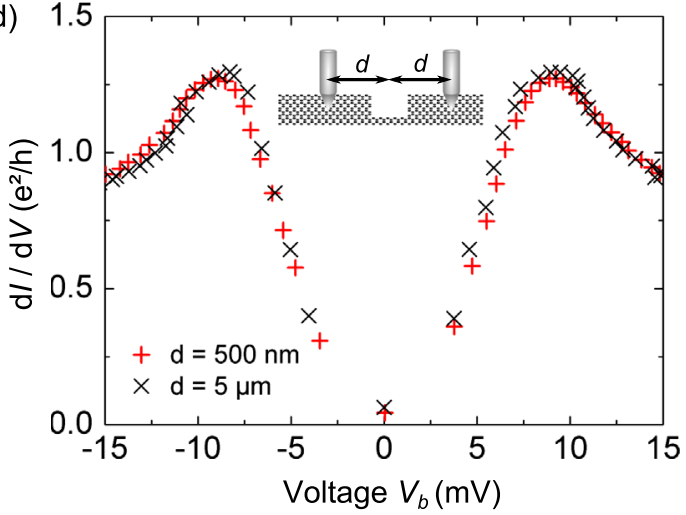

FIG. 3. Electronic transport across GNCs. (a) $I V$ curves across a GNC with length $L=6 \mathrm{~nm}$ and width $W=2 \mathrm{~nm}$ for temperatures between $T=28$ and $300 \mathrm{~K}$. The solid lines indicate corresponding fits to the Kaiser expression [40]. The violet curve is the $I V$ curve of the pristine ribbon, prior to STM lithography. (b) Differential conductance of the $I V$ curve shown in (a). Conductance peaks $G_{P}$ at voltage $V_{P}$ are clearly visible for the complete temperature range. The violet curve is the differential conductance of the pristine ribbon. (c) Peak conductances $G_{P}$ and peak voltages $V_{P}$ extracted from differential conductance curves. The peak conductance is exponentially decreasing with increasing temperature while the voltage position of the peak remains constant. (d) Differential conductance across a GNC measured with two different contact spacings $d=500 \mathrm{~nm}$ and $5 \mu \mathrm{m}$. The inset depicts the arrangement of the probes on both sides of the constriction.

each other (cf. Ref. [25], Fig. 3) and are not distinguished in the following. In order to avoid Joule heating in these constrictions most of the experiments were performed with current densities not exceeding $10^{8} \mathrm{~A} / \mathrm{cm}^{2}$. The differential conductance curves of the sidewall GNC clearly indicate the opening of a transport gap around zero bias accompanied by conductance peaks located symmetrically at about $\pm 9 \mathrm{mV}$. The positions of the conductance peaks $\left(V_{P}\right)$ remain almost constant throughout the whole temperature range and show only a slight decrease in the low temperature regime. The conductance peaks are most prominent at low temperatures and decrease with increasing $T$ in an exponential manner, $G_{\text {peak }} \sim \exp \left(-k_{B} T / e V_{0}\right)$, as shown in Fig. 3(c). However, the peaks remain visible even up to room temperature. The maximum peak value is reached at the lowest attainable temperature $T=28 \mathrm{~K}$ with $G_{P} \approx 1.6 e^{2} / h$. The peak conductance is significantly higher than the conductance of the pristine ribbon, which indicates the opening of a second transport channel or the restoring of spin degeneracy in the whole ribbon.
This result is surprising since dual channel transport was found in sidewall GNRs only for contact spacings well below $500 \mathrm{~nm}[3,25,32-36]$. Here, we used contact spacings $d \geq 500 \mathrm{~nm}$ and hence expect no contribution from the second transport channel. The characteristic shape of the $d I / d V$ curve originates solely from the constriction itself and is independent of the contact spacing. This can be directly seen from Fig. 3(d) where two conductance curves for two different contact spacings $(d=500 \mathrm{~nm}$ and $d=5 \mu \mathrm{m}$ ) are displayed. The contact spacing has no influence either on the occurrence or amplitude of the conductance peak or the opening of a transport gap.

In order to explain the opening of a transport gap as well as the origin of the conductance peaks, the GNC can be viewed as a diffraction barrier. Electronic diffraction gives rise to localized currents through the constriction, which subsequently lead to transmission resonances $[5,41]$. In analogy to subwavelength optics, the whole system can be treated as a Fabry-Perot cavity [4]. For a more quantitative analysis, we calculate the bias dependence 
of the differential conductance through a GNC with a propagating edge state. In this way, we model the GNC system by a third nearest neighbor tight binding model using a recursive Green's function approach within the common Landauer-Büttiker formulation (see Refs. [25-31], Sec. IV of Supplemental Material, where also effects due to disorder are discussed). Figure 4(a) shows a typical result for a constriction with dimensions $L \approx 1 \mathrm{~nm}$ and $W \approx 1.7 \mathrm{~nm}$ at various temperatures. Pronounced conduction peaks are found in the simulations, resembling nicely the experimental findings. Thereby, the interpretation that the constrictions give rise to resonance phenomena is strongly supported. With increasing temperature we notice that the peaks broaden and the peak heights decrease in a similar manner as observed experimentally. We also want to emphasize that for our GNC configuration the resonances originate from the zeroth mode of the zigzag topology of the ribbons; thus,
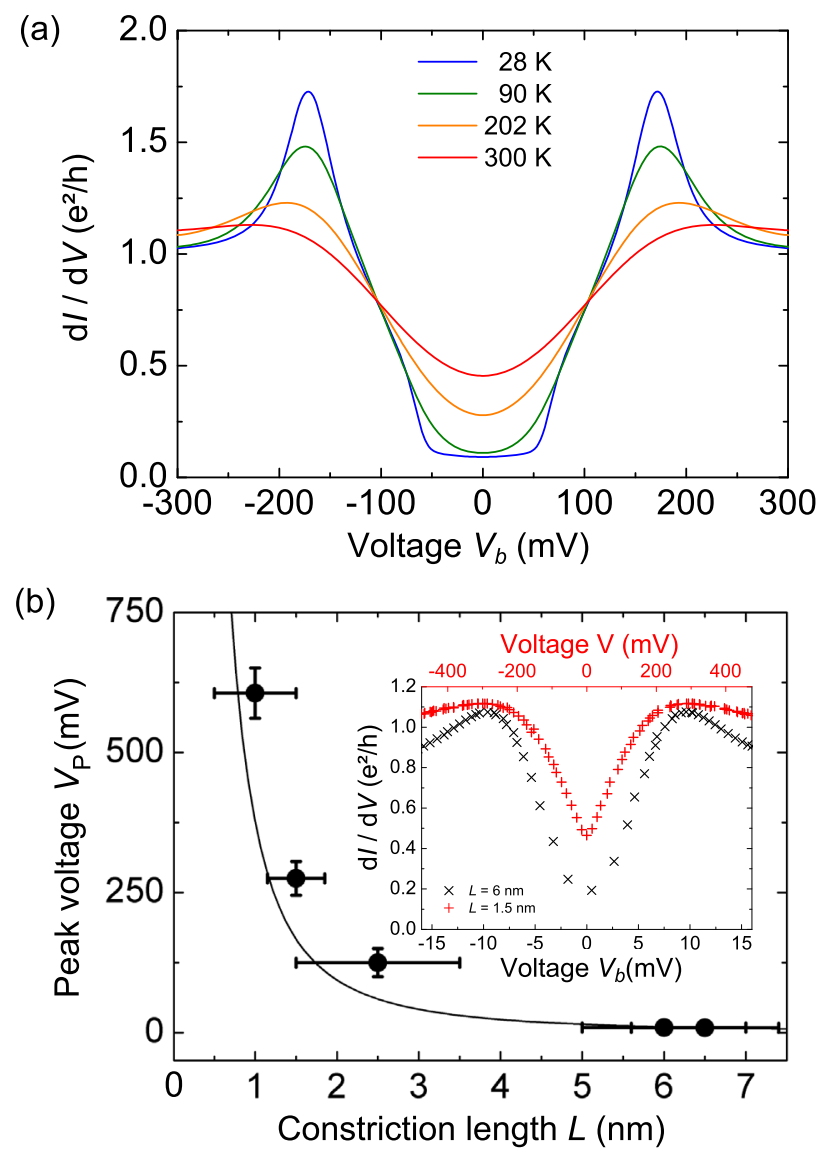

FIG. 4. Theoretical transport characteristics of GNCs and comparison with the experiment. (a) Theoretical differential conductance of a GNC of dimension $L \approx 1 \mathrm{~nm}$ and $W \approx$ $1.7 \mathrm{~nm}$ for different temperatures. (b) Voltage position of the conductance peaks plotted against the length $L$ of the constriction. The solid black line indicates the energy of the ground state in the standard particle-in-a-box picture. Inset: measured conductance at $T=298 \mathrm{~K}$ for constriction lengths $L \approx 6 \mathrm{~nm}$ (black) and $L \approx 1.5 \mathrm{~nm}$ (red). Note the different voltage scales for the two curves. mode coupling effects, e.g., coming along with antiresonances like seen in wider 2DEG wire systems, are not pivotal in our case within the small energy window [42].

The edge state gives rise to the constant $1 e^{2} / h$ conductance regime for $V>V_{P}$, whereas the peak features are caused by resonances within the constriction. The energy of the resonance is highly dependent on the length of the constriction. which can be understood in terms of a simple particle-in-a-box picture. Indeed, the resonance energy follows accurately the scaling law $E \propto 1 / L^{2}$ as shown in Fig. 4(b). While the voltage position of the resonance peak shifts drastically upon changing the dimensions of the constriction, the general shape of the differential conductance remains almost unaltered [cf. the inset of Fig. 4(b) where the $d I / d V$ curves at $T=298 \mathrm{~K}$ are shown for constrictions of length $L \approx 6 \mathrm{~nm}$ (black) and $L \approx 1.5 \mathrm{~nm}$ (red)]. This indicates that the underlying physical mechanism that gives rise to the resonance peaks is the same for narrow and wide constrictions.

These results show that graphene nanoconstrictions can be effectively used as electronic diffraction barriers. GNRs on the sidewalls of SiC mesa structures are an ideal template for this purpose. STM lithography allows us to define constrictions in situ with variable dimensions of only a few nanometers. The excellent structural and electronic quality of the self-assembled ribbons as well as the subsequently defined constrictions give rise to electron interference phenomena. Direct probing with local transport reveals the emergence of conductance peaks and transport gaps, which can serve as a hallmark for electron interference. The stability of these features up to room temperature opens up the possibility to use them in novel electronic nanodevices. Sidewall GNRs can serve as connectors between multiple constrictions as well as to the contacts over distances of several micrometers due to their exceptional transport properties. Hence, such devices would solely rely on the photonlike nature of electrons in graphene and belong to a new class of fully coherent electronics.

Financial support by the Deutsche Forschungsgemeinschaft is gratefully acknowledged by J. B., J. A. and C. T. The Center for Nanostructured Graphene (CNG) is sponsored by the Danish National Research Foundation, Project No. DNRF103.

*tegenkamp@fkp.uni-hannover.de

[1] A. S. Mayorov et al., Nano Lett. 11, 2396 (2011).

[2] C. Berger et al., Science 312, 1191 (2006).

[3] J. Baringhaus et al., Nature (London) 506, 349 (2014).

[4] P. Darancet, V. Olevano, and D. Mayou, Phys. Rev. Lett. 102, 136803 (2009).

[5] S. Ihnatsenka and G. Kirczenow, Phys. Rev. B 85, 121407 (2012). 
[6] A. Rycerz, J. Twozydlo, and W. J. Beenakker, Nat. Phys. 3, 172 (2007).

[7] Y. Lu, C. A. Merchant, M. Drndic, and A. T. Charlie Johnson, Nano Lett. 11, 5184 (2011).

[8] N. Tombros, A. Veligura, J. Junesch, M. H. D. Guimarães, I. J. Vera-Marun, H. T. Jonkman, and B. J. van Wees, Nat. Phys. 7, 697 (2011).

[9] B. Özyilmaz, P. Jarillo-Herrero, D. Efetov, and P. Kim, Appl. Phys. Lett. 91, 192107 (2007).

[10] K. Wakabayashi, Y. Takane, and M. Sigrist, Phys. Rev. Lett. 99, 036601 (2007).

[11] M. Y. Han, J. C. Brant, and P. Kim, Phys. Rev. Lett. 104, 056801 (2010).

[12] K. Todd, H.-T. Chou, S. Amasha, and D. GoldhaberGordon, Nano Lett. 9, 416 (2009).

[13] J. Baringhaus, J. Aprojanz, J. Wiegand, D. Laube, M. Halbauer, J. Hübner, M. Oestreich, and C. Tegenkamp, Appl. Phys. Lett. 106, 043109 (2015).

[14] I. Palacio et al., Nano Lett. 15, 182 (2015).

[15] J. Baringhaus, F. Edler, and C. Tegenkamp, J. Phys. Condens. Matter 25, 392001 (2013).

[16] M. Sprinkle, M. Ruan, Y. Hu, J. Hankinson, M. Rubio-Roy, B. Zhang, X. Wu, C. Berger, and W. A. de Heer, Nat. Nanotechnol. 5, 727 (2010).

[17] W. A. de Heer, C. Berger, M. Ruan, M. Sprinkle, X. Li, Y. $\mathrm{Hu}, \mathrm{B}$. Zhang, J. Hankinson, and E. Conrad, Proc. Natl. Acad. Sci. U.S.A. 108, 16900 (2011).

[18] M. S. Nevius, F. Wang, C. Mathieu, N. Barrett, A. Sala, T. O. Menteş, A. Locatelli, and E. H. Conrad, Nano Lett. 14, 6080 (2014).

[19] J. Hicks et al., Nat. Phys. 9, 49 (2013).

[20] L. Tapaszto, G. Dobrik, P. Lambin, and L. P. Biro, Nat. Nanotechnol. 3, 397 (2008).

[21] G. Z. Magda, X. Jin, I. Hagymási, P. Vancsó, Z. Osváth, P. Nemes-Incze, C. Hwang, L. P. Biró, and L. Tapasztó, Nature (London) 514, 608 (2014).

[22] X.Z. Yu, C. G. Hwang, C. M. Jozwiak, A. Köhl, A. K. Schmid, and A. Lanzara, J. Electron Spectrosc. Relat. Phenom. 184, 100 (2011).

[23] J. Baringhaus, F. Edler, C. Neumann, C. Stampfer, S. Forti, U. Starke, and C. Tegenkamp, Appl. Phys. Lett. 103, 111604 (2013).
[24] M. Settnes, S. R. Power, D. H. Petersen, and A.-P. Jauho, Phys. Rev. Lett. 112, 096801 (2014).

[25] See Supplemental Material at http://link.aps.org/ supplemental/10.1103/PhysRevLett.116.186602, which includes Refs. [26-36], for the derivation of the equations for the theoretical calculations and further details about the ballistic channel.

[26] P.-H. Chang and B. K. Nikolic, Phys. Rev. B 86, 041406 (2012).

[27] A. Lherbier, S. M.-M. Dubois, X. Declerck, Y.-M. Niquet, S. Roche, and J.-C. Charlier, Phys. Rev. B 86, 075402 (2012).

[28] S. Datta, Electronic Transport in Mesoscopic Systems (Cambridge University Press, Cambridge, England, 1997).

[29] M. P. L Sancho, J. M. Sancho, J. M. L. Sancho, and J. Rubio, J. Phys. F 15, 851 (1985).

[30] C. H. Lewenkopf and E. Mucciolo, J. Comput. Electron. 12, 203 (2013).

[31] M. Settnes, S. R. Power, J. Lin, D. H. Petersen, and A.-P. Jauho, Phys. Rev. B 91, 125408 (2015).

[32] Z.-D. Chu and L. He, Sci. China Mater. 58, 677 (2015).

[33] L. F. Huang, G. R. Zhang, X. H. Zheng, P. L. Gong, T. F. Cao, and Z. Zeng, JPCM 25, 055304 (2013).

[34] W. Norimatsu and M. Kusunoki, Physica (Amsterdam) 42E, 691 (2010).

[35] Y.-W. Son, M. L. Cohen, and S. G. Louie, Nature (London) 444, 347 (2006).

[36] N. Levy, S. A. Burke, K. L. Meaker, M. Panlasigui, A. Zettl, F. Guinea, A. H. Castro Neto, and M. F. Crommie, Science 329, 544 (2010).

[37] L. C. Venema, J. W. G. Wildöer, H. L. J. Temminck Tuinstra, C. Dekker, A. G. Rinzler, and R. E. Smalley, Appl. Phys. Lett. 71, 2629 (1997).

[38] D.-H. Kim, J.-Y. Koo, and J.-J. Kim, Phys. Rev. B 68, 113406 (2003).

[39] W. Norimatsu and M. Kusunoki, Physica (Amsterdam) 42E, 691 (2010).

[40] A. B. Kaiser and Y. W. Park, Synth. Met. 152, 181 (2005).

[41] T. Gunst, J. T. Lu, P. Hedegard, and M. Brandbyge, Phys. Rev. B 88, 161401 (2013).

[42] K. Nakazato and R. J. Blaikie, J. Phys. Condens. Matter 3, 5729 (1991). 